\title{
The Limiting Temperature of hot nuclei from microscopic Equation of State
}

\author{
M. BALDO*, L. S. FERREIRA** and O.E. NICOTRA*† \\ ** Centro de Física das Int. Fund. and Dep. de Física, Inst. Sup. Tecn. \\ Av. Rovisco Pais, 1096 Lisboa, Portugal \\ * Istituto Nazionale di Fisica Nucleare, Sezione di Catania and Dipartimento di Fisica, \\ Universitá di Catania, Via S. Sofia 64, I-95123 Catania, Italy \\ † Dipartimento di Fisica, Universitá di Messina \\ Salita Sperone 31, 98166, S. Agata, Messina
}

\begin{abstract}
The limiting temperature $T_{l i m}$ of a series of nuclei is calculated employing a set of microscopic nuclear Equations of State (EoS). It is shown that the value of $T_{\text {lim }}$ is sensitive to the nuclear matter Equation of State used. Comparison with the values extracted in recent phenomenological analysis appears to favour a definite selection of EoS' s. On the basis of this phenomenological analysis, it seems therefore possible to check the microscopic calculations of the nuclear EoS at finite temperature, which is hardly accessible through other experimental informations.
\end{abstract}

PACS numbers: 21.65.+f, 21.30.-x, 25.70.-z, 26.50.+x, 26.60.+c

\section{INTRODUCTION}

The knowledge of the Equation of State (EoS) of nuclear matter at finite temperature is one of the fundamental issues in nuclear physics. Phenomenological information on the EoS can be obtained from experimental data on heavy ion collisions at intermediate energies and astrophysical observations on supernovae explosions and neutron stars. The nuclear matter EoS is believed to go through a liquid-gas phase transition, as many theoretical calculations indicate [1-4]. However, if this phase transition exists, does not possess a direct correspondence in finite nuclei, due to the presence of the Coulomb and finite size effects. In particular, the Coulomb interaction is of long range and strong enough to modify the nature of the phase transition. Instead, it has been recognized by some authors $[5,6]$, that the nuclear EoS is related to the maximal temperature a nucleus can sustain before reaching mechanical instability. This "limiting temperature" $T_{l i m}$ is mainly the maximal temperature at which a nucleus can be observed.

It has to be stressed that the reaction dynamics can prevent the formation of a true compound nucleus. The onset of incomplete fusion reactions can mask completely the possible presence of fusion or nearly fusion processes. At higher energies, the heavy ion reaction can be fast enough that no (nearly) thermodynamical equilibrium can be reached, as demanded 
in a genuine standard fusion-evaporation reaction. However, combined theoretical and experimental analysis [7] indicate that a nearly equilibrium condition is reached in properly selected multifragmentation heavy ion reactions at intermediate energy. The main experimental observation is the presence of a plateau in the so-called "caloric curve", i.e. in the plot of temperature vs. total excitation energy [8-11]. This behaviour was qualitatively predicted by the Copenhagen statistical model [12] of nuclear multifragmentation. The relation between multifragmentation processes and the nuclear EoS was extensively studied by several authors within the statistical approach to heavy ion reaction at intermediate energy [13-19].

In different experiments, various methods are used to extract from the data the values of the temperature of the source which produces the observed fragments, but a careful analysis of the data [7] seems to indicate a satisfactory consistency of the results. In refs. [7,20] an extensive set of experimental data was analyzed and it was shown that the temperature at which the plateau starts is decreasing with increasing mass of the residual nucleus which is supposed to undergo fragmentation. Both the values and the decreasing trend of this temperature turn out to be consistent with its interpretation as limiting temperature $T_{\text {lim }}$. According to this interpretation, at increasing excitation energy the point where the temperature plot deviates from Fermi gas behaviour and the starting point of the plateau mark the critical point for mechanical instability and the onset of the multifragmentation regime. The corresponding value of the critical temperature can be calculated within the droplet model, and indeed many estimates based on Skyrme forces are in fairly good agreements with the values extracted from phenomenology [7,6]. Moreover, the relation between nuclear matter critical temperature $T_{c}$ and $T_{\text {lim }}$ appears to be quite stable and independent on the particular EoS and method used, which allows [20] to estimate $T_{c}$ from the set of values of $T_{\text {lim }}$.

In general, one can expect that $T_{\text {lim }}$ is substantially smaller than the critical one, $T_{c}$. In fact, both the Coulomb repulsion and the lowering of the surface tension with increasing temperature tend to destabilize the nucleus with respect to infinite nuclear matter. Since the surface tension goes to zero at the critical temperature, $T_{\text {lim }}$ is reached much before $T_{c}$. These predictions were checked in the seminal paper of ref. [5], as well as in further studies based on macroscopic Skyrme forces [6], for which a simple relationship was established between $T_{l i m}$ and $T_{c}$. In ref. [21] it was shown, however, that if microscopic EoS are used, the relationship between $T_{\text {lim }}$ and $T_{c}$ is not so simple and systematic as in the case of Skyrme force EoS, and only a qualitative connection exists.

In this paper we consider the finite temperature EoS in the framework of microscopic non-relativistic and relativistic many-body theory of nuclear matter and the corresponding critical temperature. Then the limiting temperature for finite nuclei is calculated on the basis of the corresponding EoS. The comparison with phenomenology shows the sensitivity of $T_{\text {lim }}$ to the microscopic EoS. These results open the possibility of a direct check of the microscopic theory of the nuclear matter EoS. Indeed, all the considered microscopic EoS reproduce the empirical saturation point, but their behaviour at finite temperature can be quite different. 


\section{THE MICROSCOPIC EOS}

Microscopic calculations of the nuclear EoS at finite temperature are quite few. The variational calculation by Friedman and Pandharipande [1] was one of the first few semimicroscopic investigation of the finite temperature EoS. The results predict a liquid-gas phase transition, with a critical temperature $T_{c}=18-20 \mathrm{MeV}$. Later, Brueckner calculations at finite temperature [2] confirmed these findings with very similar values of $T_{c}$.

The Van der Waals behaviour, which leads to the liquid-gas phase transition, was also found in the finite temperature relativistic Dirac-Brueckner (DB) calculations of ref. [4,3]. A liquid-gas phase transition was clearly observed, but at a much lower value, $T_{c} \approx 10 \mathrm{MeV}$. It seems unlikely that such lower critical temperature can be attributed to relativistic effects, since the critical density is a fraction of the saturation one, where relativistic effects are expected to play no role. It is more likely that this lower value of $T_{c}$ is due to the smaller value of the effective mass, and we will present evidence of that later.

More recently, chiral perturbation theory at finite temperature was used [22] to calculate the nuclear matter EoS, up to three-loop level of approximation. The theory is a low density expansion, and it appears appropriate to study the critical point, where the density is a fraction of the saturation density. Again a Van der Waals behaviour was found, with a critical temperature $T_{c} \approx 25 \mathrm{MeV}$.

This set of nuclear matter EoS can be considered representative of the possible predictions from microscopic many-body theory. Here in the sequel of this section we will remind briefly the non-relativistic Bloch and De Dominicis formalism, used in our calculations, which is an extension to finite temperature of the Bethe-Brueckner-Goldstone (BBG) expansion. The formalism used in Dirac-Brueckner calculations at finite temperature is formally very similar, as we will discuss later. For the chiral perturbation the formalism is of course quite different, and we refer the reader to the original paper [22].

The finite temperature Bloch and De Dominicis linked diagram expansion is based on the Grand-canonical representation and has the property to lead, in the zero temperature limit, to the BBG expansion of the ground state energy. The grand canonical potential per particle $\omega$ is written as the sum of the unperturbed potential $\omega_{0}^{\prime}$ and a correlation term $\Delta \omega$,

$$
\omega=\omega_{0}^{\prime}+\Delta \omega
$$

corresponding to the one-body grand canonical potential, and a power series expansion in the interaction $H_{1}$ involving connected diagrams only, respectively. The unperturbed potential is defined by,

$$
\omega_{0}^{\prime}=\omega_{0}-\sum_{k} U_{k} n(k)
$$

with $n(k)$ the finite temperature Fermi distribution, $\omega_{0}$ the grand canonical potential of the independent particle hamiltonian $H_{0}^{\prime}$, and the summation over the single particle potential $U_{k}$ represents the first potential insertion diagram [2]. Therefore, $\omega_{0}^{\prime}$ includes all one-body contributions and its explicit form reads

$$
\begin{gathered}
\omega_{0}^{\prime}=-\frac{2}{\pi^{2}} \int_{0}^{+\infty} k^{2} d k\left[\frac{1}{\beta} \log \left(1+e^{-\beta\left(e_{k} \mu\right)}\right)\right. \\
+U(k) n(k)]
\end{gathered}
$$


$\mu$ being the chemical potential, and

$$
\begin{aligned}
\Delta \omega=\frac{2}{(2 \pi)^{3}} \sum_{l S J T} \hat{J}^{2} \hat{T}^{2} \int d q \int P^{2} d P e^{-\beta\left(\bar{E}_{P q}-2 \mu\right)} & \\
& \cdot d(q, P) \arctan \left[\frac{\pi\left(q l\left|\mathcal{K}^{S J T}\left(\bar{E}_{P q}\right)\right| q l\right) q^{2} \bar{Q}(q, P)}{d(q, P)}\right],
\end{aligned}
$$

where the density of state $d$ is given by,

$$
d(q, P)=\left|\frac{\partial \bar{E}_{q P}}{\partial q}\right|=\left|\frac{2 \hbar^{2} q}{m}+\frac{\partial}{\partial q} \bar{U}_{q P}\right| .
$$

The two-particle energy $\bar{E}_{q P}$, the Pauli operator $\bar{Q}_{q P}$ and the potential felt by two particle $\bar{U}_{q P}$, are all angle averaged quantities [2]. These angular averaging is expected to be accurate, allowing us to make the contribution of different channels additive, since then, only the diagonal part of the finite temperature scattering matrix $K$ contributes. The quantum numbers $l S J T$ specify the two-body channel and $\hat{A}=\sqrt{2 A+1}$.

The single particle potential and the two-body scattering matrix $K$ satisfy the selfconsistent equations

$$
U\left(\mathbf{k}_{1}\right)=\sum_{\sigma \tau} \sum_{\mathbf{k}_{2}}\left\langle k_{1} k_{2}|K(\omega)| k_{1} k_{2}\right\rangle_{A} n\left(k_{2}\right)
$$

and

$$
\begin{aligned}
& \left\langle k_{1} k_{2}|K(\omega)| k_{3} k_{4}\right\rangle=\left\langle k_{1} k_{2}|v| k_{3} k_{4}\right\rangle+ \\
& \sum_{k_{3}^{\prime} k_{4}^{\prime}}\left\langle k_{1} k_{2}|v| k_{3}^{\prime} k_{4}^{\prime}\right\rangle \frac{n_{>}\left(k_{3}^{\prime}\right) n_{>}\left(k_{4}^{\prime}\right)}{\omega-e}\left\langle k_{3}^{\prime} k_{4}^{\prime}|K(\omega)| k_{3} k_{4}\right\rangle .
\end{aligned}
$$

In Eq. (4)

$$
\begin{aligned}
& \left\langle k_{1} k_{2}|\mathcal{K}(\omega)| k_{3} k_{4}\right\rangle= \\
& \quad\left(n_{>}\left(k_{1}\right) n_{>}\left(k_{2}\right) n_{>}\left(k_{3}\right) n_{>}\left(k_{4}\right)\right)^{\frac{1}{2}}\left\langle k_{1} k_{2}|K(\omega)| k_{3} k_{4}\right\rangle
\end{aligned}
$$

In all the previous equations $\omega=E_{k_{1}}+E_{k_{2}}, e=E_{k_{3}^{\prime}}+E_{k_{4}^{\prime}}$, with $E_{k}=\hbar^{2} k^{2} / 2 m+U_{k}$. Eq. (7) coincides with the Brueckner equation for the Brueckner $G$ matrix at zero temperature, if the single particle occupation number $n(k)$ are taken at $T=0$. At finite temperature $n(k)$ is a Fermi distribution. In Eqs. $(7,8) n_{>}(k)=1-n(k)$. It has to be noticed, that only the principal part has to be considered in the integration, thus making $K$ a real matrix.

Eqs. 6 and 7 have to be solved self-consistently for the single particle potential. For a given density and temperature we solve the self-consistent equations along with the Eq. (9) for the chemical potential $\tilde{\mu}$,

$$
\rho=\sum_{k} n(k)=\sum_{k} \frac{1}{e^{\beta\left(E_{k}-\tilde{\mu}\right)}+1}
$$


Then we obtain the grand canonical potential $\omega$ from Eq. (4). Finally we extract the free energy per particle $f$ from the relation,

$$
f=\omega \rho+\tilde{\mu} .
$$

The pressure $p$ is calculated performing a numerical derivative of $f$, i.e. $p=\rho^{2} \partial f / \partial \rho$. Notice that the chemical potential $\tilde{\mu}$ extracted from Eq. (9) does not coincide with the exact thermodynamical chemical potential $\mu$ given by

$$
\mu=\frac{\partial F}{\partial N}=f+\rho\left(\frac{\partial f}{\partial \rho}\right)
$$

which is the one actually adopted, in order to satisfy the Hugenholtz-Van Hove theorem [2].

It turns out that [2] the dominant diagrams in the expansion are the ones that correspond to the zero temperature BBG diagrams, where the temperature is introduced in the occupation numbers only, represented by Fermi distributions, thus justifying this commonly used procedure of naively introducing the temperature effect.

The same prescription has been used in Dirac-Brueckner calculations. The formalism is therefore in principle very similar.

\section{THE LIMITING TEMPERATURE OF FINITE NUCLEI}

Following ref. [5] the limiting temperature can be evaluated within the liquid drop model, which should be accurate enough for medium-heavy nuclei. The nucleus is described in terms of a droplet surrounded by a vapour, in thermal and mechanical equilibrium. In the model one adds to the droplet pressure and chemical potential the contributions due to the Coulomb force and surface tension, which are evaluated assuming a spherical droplet. These additional terms read,

$$
\begin{aligned}
\delta P & =P_{C}+P_{S}=\left(\frac{Z^{2} e^{2}}{5 A} \rho-2 \alpha(T)\right) / R \\
\delta \mu & =\frac{6 Z^{2} e^{2}}{5 A R}
\end{aligned}
$$

where $R$ is the droplet radius $R=\left(\frac{3 A}{4 \pi \rho}\right)^{1 / 3}, \rho$ is the droplet density and for $\alpha(T)=\alpha_{0}(1+$ $\left.\frac{3}{2} T / T_{c}\right)\left(1-T / T_{c}\right)^{3 / 2}$, with $T_{c}=20 \mathrm{MeV}$ the nuclear matter critical temperature and the surface tension at zero temperature $\alpha_{0}=1.14 \mathrm{MeV} \mathrm{fm}^{-2}$, obtained from the semi-empirical mass formula. The Coulomb interaction introduces and additional positive pressure $P_{C}$ and a repulsive contribution to the bulk chemical potential $\mu$, while the surface tension provides and additional negative pressure term which tends to stabilize the system. At increasing temperature the surface tension decreases and the system becomes unstable against Coulomb dissociation. The simplest way to observe the modifications introduced by these terms is to consider the plot of the chemical potential as a function of pressure, both for nuclear matter and for the droplet model.

The intersection between the liquid and the vapour branches defines the coexistence point in nuclear matter. The additional terms will only shift the liquid branch, since the vapour is assumed to be uniform and uncharged, leading to a new coexistence point. 
This procedure was followed for the set of nuclear matter EoS discussed in the previous section. At the lowest densities in the vapour region, needed in the calculations, the microscopic EoS was extended following ref. [2].

\section{RESULTS AND DISCUSSION}

To illustrate the procedure followed in the microscopic calculations of EoS and $T_{\text {lim }}$ in the framework of many-body theory, the nuclear matter free energy is reported in Fig. 1a as a function of density for various temperature in the case of the Bonn B potential [23]. The points indicates the actual microscopic calculations, the full lines the corresponding polynomial fits. The figure illustrates the precision and stability of the numerical procedure. The three-body force, discussed in [2], was included with adjusted parameters to reproduce the correct saturation point. From the free energy, by numerical derivative, one gets the pressure depicted in Fig. 1b. The critical temperature for the liquid-gas phase transition is the lowest temperature for which the isotherm is monotonic and the critical point is the corresponding inflexion point on the isotherm. From Fig. 1b the critical temperature appears to be around $T_{c} \approx 18 \mathrm{MeV}$, slightly below the value obtained in ref. [2] for the Argonne $\mathrm{v}_{14}$ potential [24] ( $\left.T_{c} \approx 20 \mathrm{MeV}\right)$. This shows that there is some sensitivity of $T_{c}$ on the NN interaction. It has to be stressed that the two EoS have very close saturation points.

As it is well known, the Dirac-Brueckner approach gives in general a better saturation point than the conventional Brueckner calculations (without three-body force). It has been shown that this is mainly due to the modification of the nucleon Dirac spinor inside nuclear matter, which can be described by the contribution of the so-called Z-diagram [25], corresponding to the virtual creation of a nucleon-antinucleon pair. The Z-diagram can be viewed as a particular three-body force, which is repulsive at all densities. The density dependence of this contribution was studied in ref. [25] and was found to be of the type $\Delta e=C \rho^{8 / 3}$, with the coefficient $C$ depending on the $\mathrm{NN}$ interaction. In ref. [23] it was found that such a term can account very precisely for the difference between the Dirac-Brueckner calculation and the corresponding non-relativistic Brueckner one.

Finite temperature Dirac-Brueckner calculations are quite few in the literature $[3,4]$. Furthermore, for our analysis we need the free energy as a function of density at small steps of the temperature. Fortunately it is possible to estimate accurately the temperature dependence of the free energy at a given density by a simplified procedure, avoiding the complexity of the full finite temperature Dirac-Brueckner calculations. Once the zero temperature EoS is known, we assume that the free energy at $T \neq 0$ can be obtained by including the variations of both entropy and internal energy of a free Fermi gas with the value of the effective mass ( at $k=k_{F}$ ) equal to the one calculated at the same density and at $T=0$. In this way one neglects the variation with temperature of the effective mass and of the interaction energy. Both these variations turn out to be small at the Brueckner level [2], and indeed the same procedure applied to to non-relativistic Brueckner calculations give excellent agreement with the full calculations [2].

We applied this procedure to the EoS of ref. [3], by fitting the Dirac-Brueckner EoS at $T=0$ and calculating the free energy at finite temperature from the corresponding effective mass. At variance with the previous calculations of ref. [2], we preferred here to fit directly 
the EoS at zero temperature instead of applying the relativistic correction due to Z-diagram mentioned above. This should avoid any possible bias from the NN interaction. In any case, the final results are quite similar to the previous calculations. We found a critical temperature $T_{c} \approx 12 \mathrm{MeV}$, in comparison with the value of $10 \mathrm{MeV}$ reported in ref. [3]. This reasonable agreement is a further check of the simplified procedure adopted. Since the limiting temperature $T_{l i m}$ is expected to be a small fraction of the critical temperature $T_{c}$, the error introduced by the simplified procedure can be considered small enough for an accurate treatment of the Dirac-Brueckner case.

In DB calculations the single particle energy $E_{k}$ is written as [23]

$$
E_{k}=\sqrt{M^{* 2}+k^{2}}+U_{V} \quad, \quad M^{*}=M+U_{S}
$$

where $U_{S}$ and $U_{V}$ are the scalar and vector single particle potentials respectively. In the non-relativistic limit the square root is expanded in power of $k / M^{*}$. If one neglects the momentum dependence of the scalar and vector potentials, $M^{*}$ can be identified with the non-relativistic effective mass to be used in the finite temperature calculations for the Fermi gas model. In the region of the liquid-gas phase transition the non-relativistic expansion is fully justified. This is equivalent to a parabolic approximation for the single particle energy. This procedure results in values of the effective mass which are substantially smaller than in the conventional non-relativistic Brueckner calculations [23], where no parabolic approximation for the single particle potential is used [26].

For the EoS calculated within chiral perturbation theory, all the expressions are semianalytical and the whole procedure is much simpler.

Plots of the chemical potential as a function of pressure for nuclear matter are reported in Fig. 2 . The intersection between the liquid and the vapour branches defines the coexistence point in nuclear matter. Increasing the temperature, the curve shrinks and should collapse to a point at $T_{c}$, which can be thus determined in this way. The values extracted along this procedure are in good agreement with the values obtained from the plot of pressure vs. density, Fig. 1b. This illustrates the consistency and precision of the numerical procedure.

For the droplet model, including the corrections of Eq. (12), the new liquid branch, indicated by the dashed lines in Fig. 2, shows a shift with respect to nuclear matter. At low enough temperature an intersection between the liquid and vapour branches still occurs, which corresponds to the coexistence point between the liquid droplet and the nuclear matter vapour and assures that the droplet is stable. Increasing the temperature, the curve shrinks and well below $T_{c}$ it is possible to find a temperature for which the intersection between the liquid droplet and the vapour branches just disappears, as indeed reported in Fig. 2. This determines $T_{\text {lim }}$.

The droplet-vapour coexistent point, and consequently $T_{\text {lim }}$, depends on the mass and charge of the system.

Fig. 3 summarizes the results of the calculations, in comparison with the data obtained from the phenomenological analysis [7,20]. For completeness and for sake of comparison, also the results for the $\mathrm{Av}_{14}$ potential of ref. [2] is reported. The calculated values of the limiting temperature $T_{\text {lim }}$, for the considered set of microscopic nuclear matter EoS, show an overall trend which clearly reflect the corresponding trend for the critical temperature $T_{c}$ of each EoS. Smaller values of $T_{c}$ results in a smaller value of $T_{\text {lim }}$. 
The ratio between $T_{l i m}$ and $T_{c}$ for Skyrme forces was extensively studied in ref. [20]. It was found that this ratio is close to $1 / 3$ with a small dispersion. The microscopic EoS analyzed in Fig. 3 give values which follow closely this value, except the Dirac-Brueckner case, which gives a value closer to $1 / 4$ This could be attributed to the approximate procedure we used for this EoS, but in any case a value of $1 / 3$ would not alter the trend reported in Fig. 3.

More importantly, the comparison of the values of $T_{\text {lim }}$ from microscopic EoS with the phenomenological values emphasizes the sensitivity of $T_{\text {lim }}$ to the EoS. This comparison appears as a crucial test for any microscopic EoS. The EoS from ref. [22], as noticed by the authors, produces a too large value of the nucleon effective mass, and this is probably the reason of the too high value of $T_{c}$. In fact, a large effective mass reduces the increase with temperature of the kinetic energy and therefore of the free energy.

On the contrary, the DB results seem to indicate that the corresponding EoS has a too small $T_{c}$. Notice that this would be very difficult to verify with other phenomenological analysis. The reason for such a small value of $T_{c}$, and therefore of a too small value of $T_{\text {lim }}$, can be attributed again to the value of the effective mass, which is smaller than in the non-relativistic case. However, other characteristic of the EoS could play a role, like the values of the chemical potential or of the compressibility at low density (i.e. in the gas phase).

The non-relativistic BHF results appear to agree quite closely with the phenomenological values. Some dependence on the NN interaction is present, but this uncertainty is within the phenomenological uncertainty. Therefore, phenomenology appears to favour this set of EoS. These results also support the interpretation of $T_{l i m}$ as the temperature for the mechanical instability and the onset of the multifragmentation regime.

\section{ACKNOWLEDGMENTS}

We thanks very much Dr. N. Kaiser for his kindness in providing us an extended numerical table of the Equation of State developed in ref. [22].

One of us ( O. E. N. ) expresses many thanks for the kind hospitality during his stay at the Centro de Física das Int. Fund. in Lisbon, where part of this work has been developed. 


\section{REFERENCES}

[1] B. Friedman and V. R. Pandharipande, Nucl. Phys. A 361, 502 (1981).

[2] M. Baldo and L.S. Ferreira, Phys. Rev. C 59, 682 (1999).

[3] B. ter Haar and R. Malfliet, Phys. Rev. Lett. 56, 1237 (1986); Phys. Rep. 149, 207 (1987).

[4] H. Huber, F. Weber and M.K. Weigel, Phys. Rev. C57, 3484 (1999)

[5] S. Levit and P. Bonche, Nucl. Phys. A437, 426 (1985).

[6] H. Q. Song and R. K. Su, Phys. Rev. C44, 2505 (1991).

[7] J.B. Natowitz, R. Wada. K. Hagel, T.Keutgen, M. Murray, A. Makeev, L. Qin, P. Smith and C. Hamilton, Phys. Rev. C65, 034618 (2002).

[8] J. Pochdzalla et al., Phys. Rev. Lett. 75, 1040 (1995).

[9] R. Wada et al., Phys. Rev. C55, 227 (1997).

[10] J. Cibor et al., Phys. Lett. B473, 29 (2000).

[11] D. Cussol et al., Nucl. Phys. A561, 298 (1993).

[12] J. Bondorf, R. Donangelo, I.N. Mishustin and H. Schulz, Nucl. Phys. A444, 460 (1985).

[13] J. Bondorf, R. Donangelo, I.N. Mishustin, C. Pethick, H. Schulz, and K. Sneppen, Phys. Lett. B150, 57 (1985) ; Nucl. Phys. A443, 321 (1985).

[14] J. Bondorf, R. Donangelo, H. Schulz, and K. Sneppen, Phys. Lett. B162, 30 (1985)

[15] A.S. Botvina et al., Nucl. Phys. A475, 663 (1987).

[16] D. Gross et al. Prog. Part. Nucl. Phys. 30, 155 (1993), and references therein.

[17] W. A. Friedman, Phys. Rev. Lett. 60, 2125 (1988).

[18] L.P Csernai and J. I. Kapusta, Phys. Rep. 131223 (1986), and references therein.

[19] J.P. Bondorf et al., Phys. Rept. 257, 133 (1995).

[20] J.B. Natowitz, K. Hagel, Y. Ma, M. Murray, L. Qin, R. Wada and J. Wang, Phys. Rev. Lett. 89, 212701 (2002).

[21] M. Baldo, Y. H. Cai, G. Giansiracusa, U. Lombardo, H. Q. Song, Phys. Lett. B340 , 13 (1994).

[22] S. Fritsch, N. Kaiser and W. Weise, Phys. Lett. B545, 73 (2002)

[23] R. Machleidt, Adv. Nucl. Phys. 19, 189 (1989).

[24] R.B. Wiringa, R.A. Smith and T.L. Ainsworth, Phys. Rev. 29C, 1207 (1984).

[25] G. E. Brown, W. Weise, G. Baym and J. Speth, Comm. Nucl. Part. Phys. 17, 39 (1987).

[26] M. Baldo and A. Fiasconaro, Phys. Lett. B491, 240 (2000). 


\section{Figure captions}

Fig. 1a - Free energy per particle as a function of Fermi momentum at different temperatures for the Bonn potential. From top to bottom the different curves correspond to temperatures $T=2,8,12,16,20,24,28 \mathrm{MeV}$. The points represent the results of the Brueckner-HartreeFock calculations at finite temperature, the curves are the corresponding polynomial fits.

Fig. 1a - Isotherms of pressure vs. Fermi momentum corresponding to the free energy plots of Fig. 1a. The sequence of temperatures is the same as in Fig. 1a (from bottom to top).

Fig. 2 - Chemical potential vs. pressure for the Bonn potential from the Brueckner-HartreeFock calculations of Figs. 1a,1b (full line) at a given temperature. The dotted line indicates the corresponding plot for the nucleus ${ }^{208} \mathrm{~Pb}$. At this temperature the nucleus starts to be unstable, see the text for details.

Fig. 3 - Limiting temperatures as a function of mass numbers for different Equation of State in comparison with the phenomenological values (open squares with error bars). 


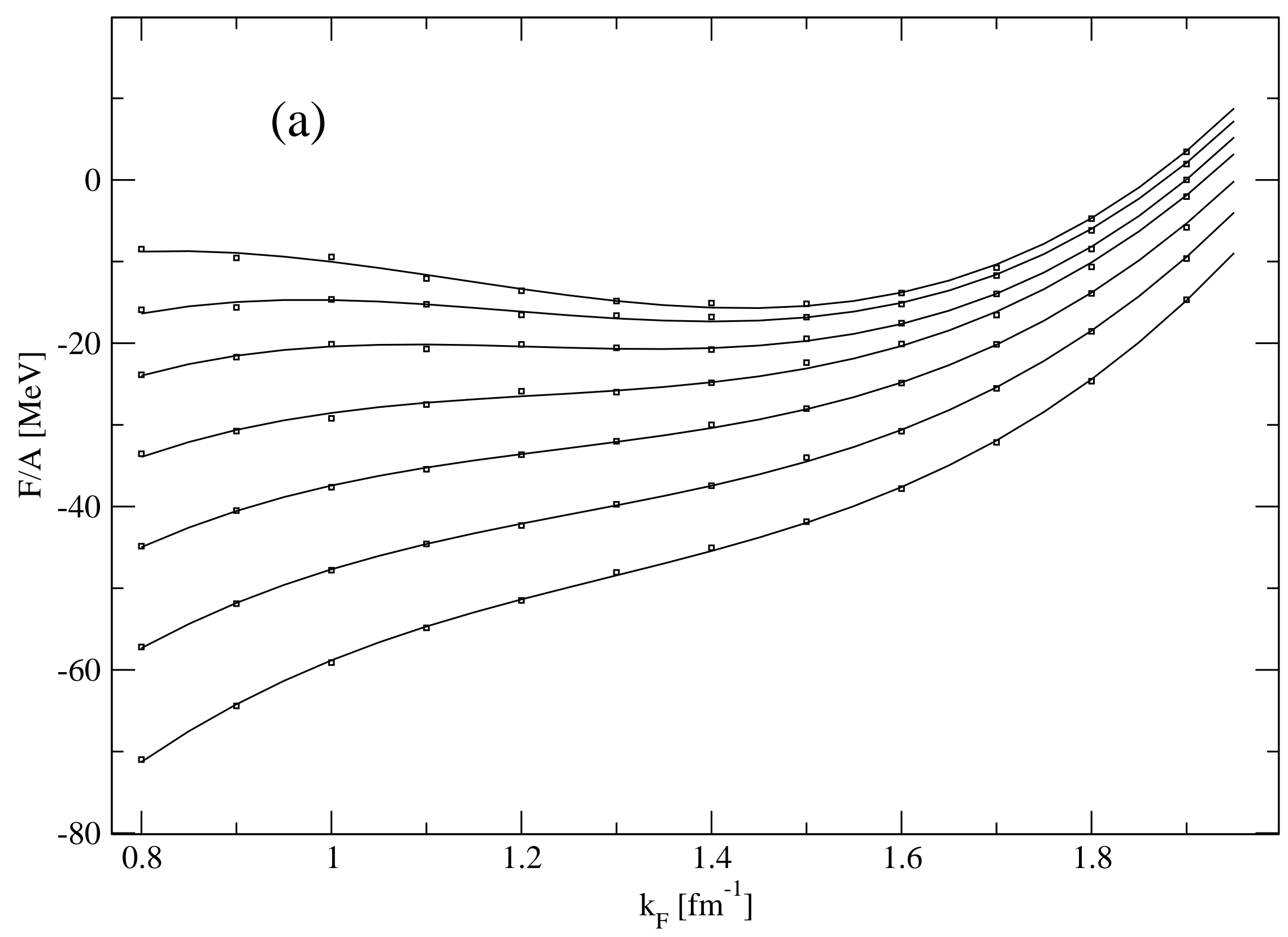




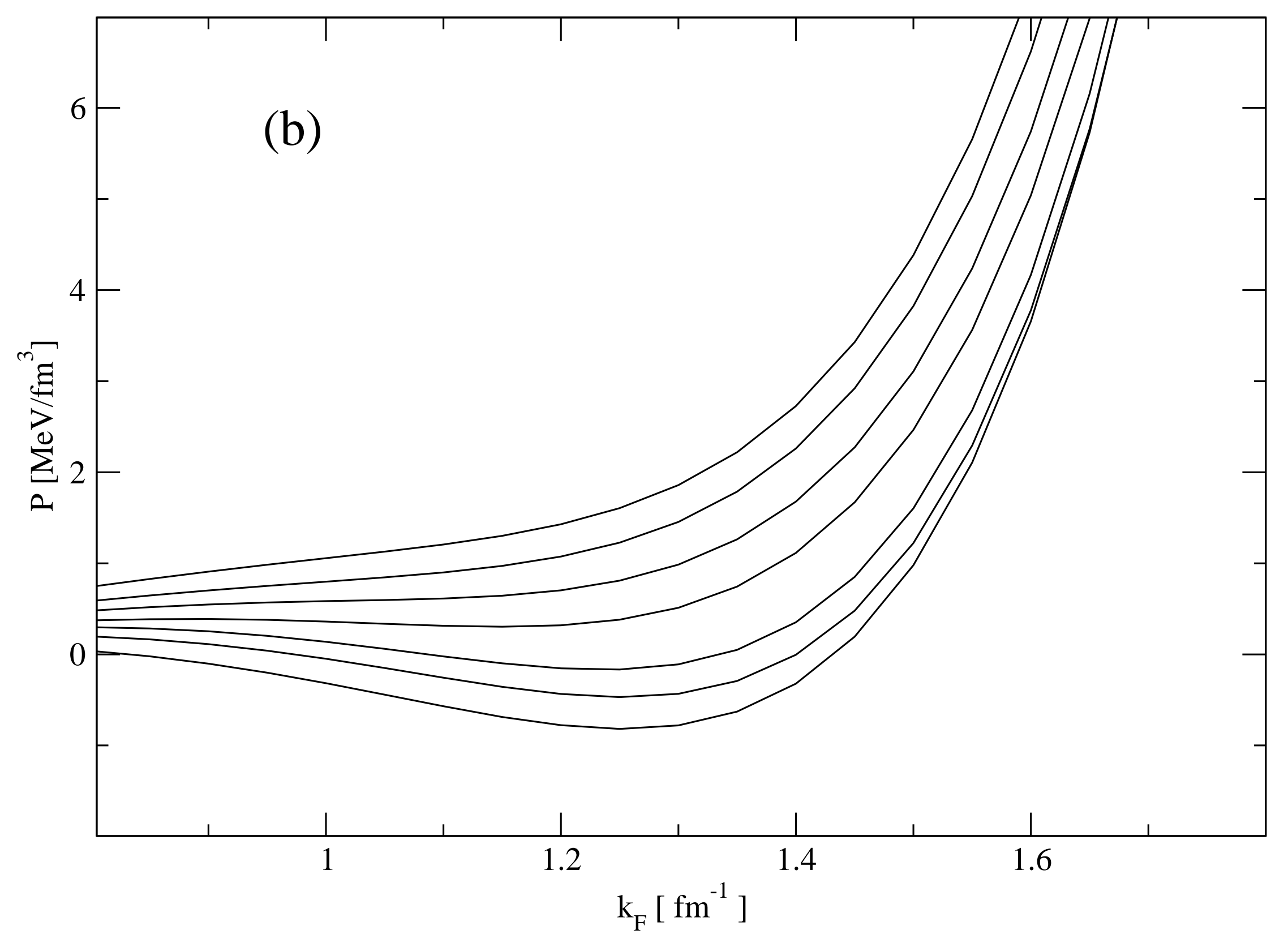




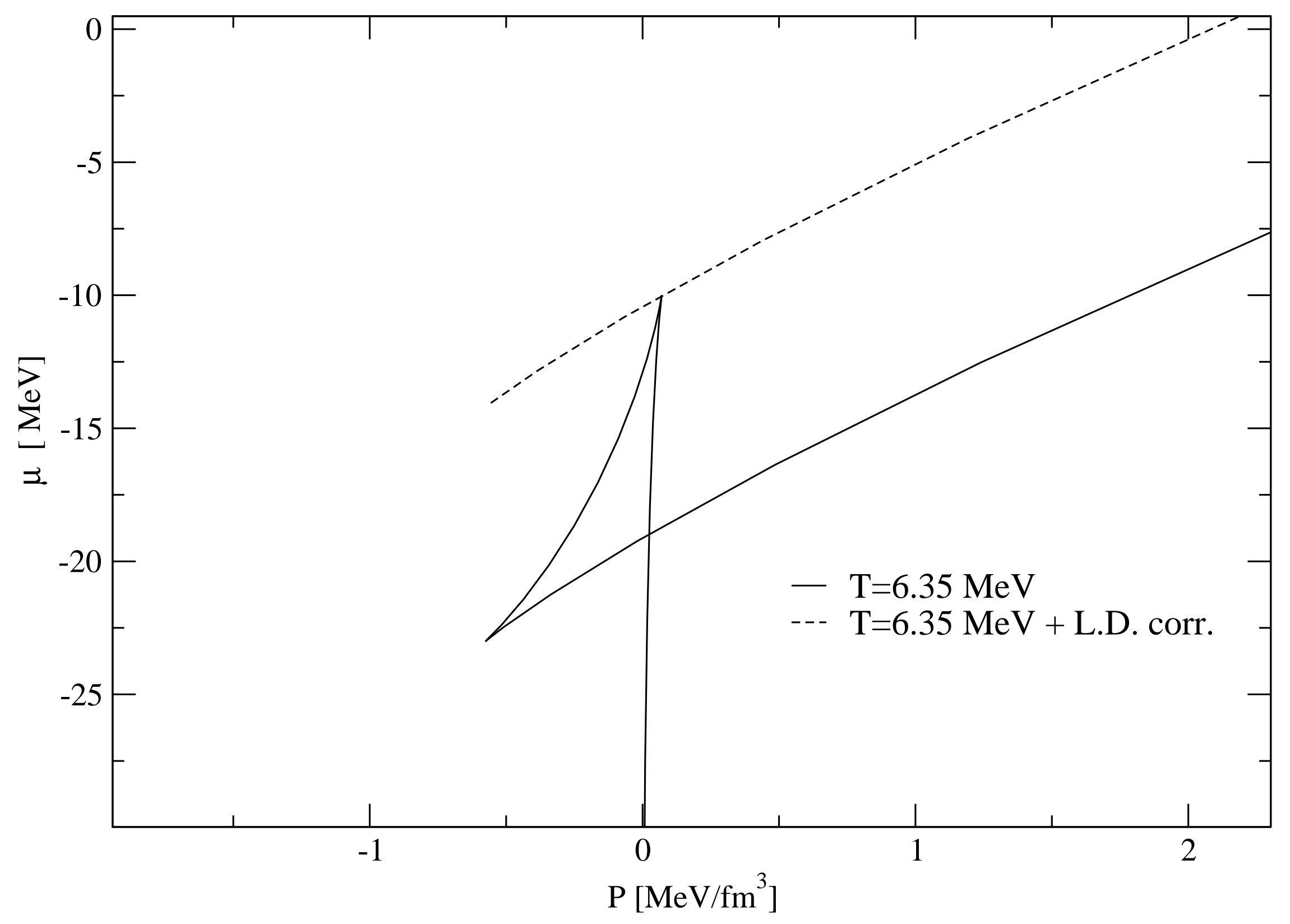




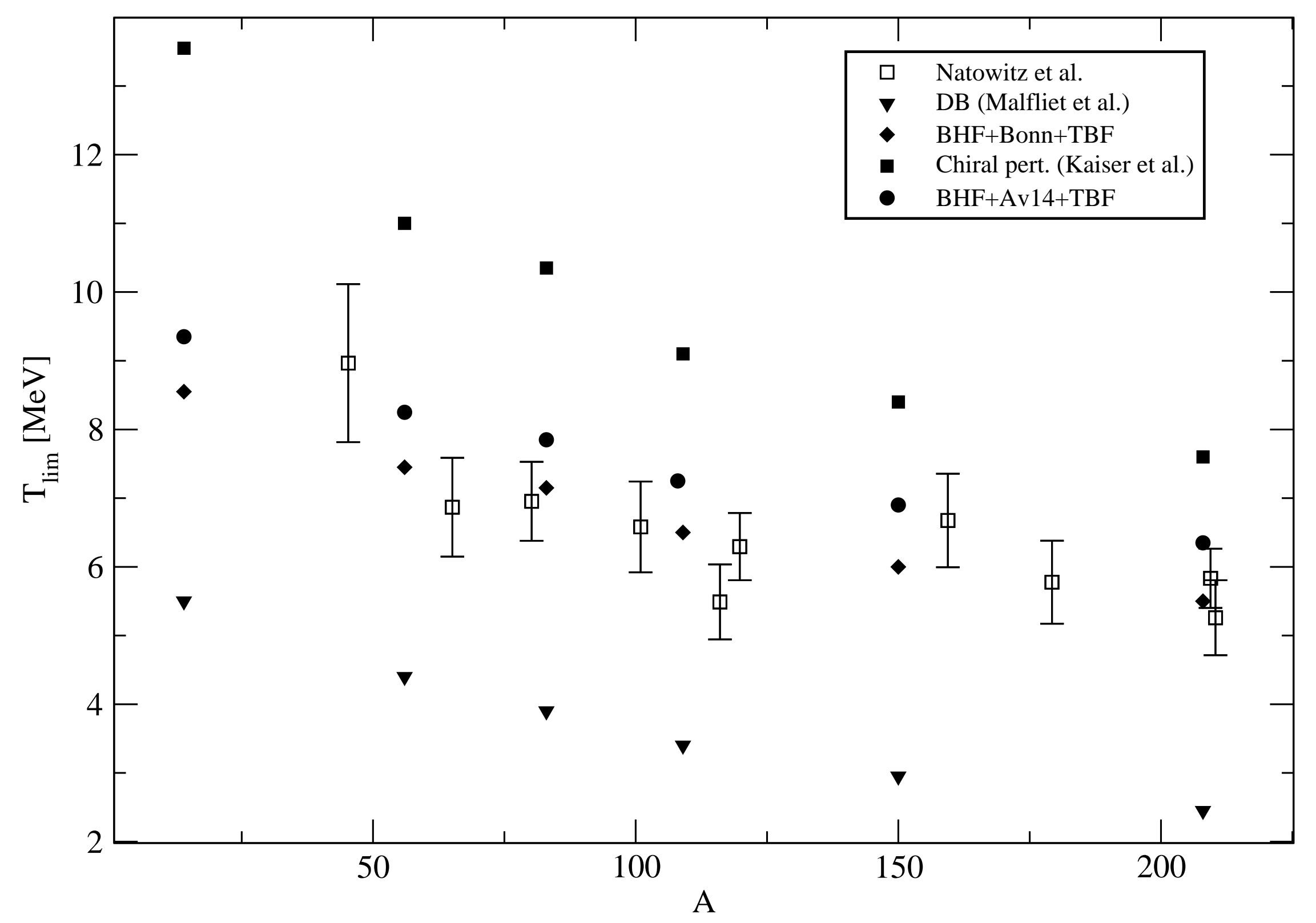

